\title{
Análisis de las narrativas de actores frente al papel de las emociones en el conflicto escolar
}

\begin{abstract}
Pág. 107 - 143
José Edilson

Barrios Leonel*

Ileana Judith

Armas Gordillo***

*Universidad

Internacional

Iberoamerica

UNINI

Ibagué- Colombia

jose21_barrios@hotmail.com

**Universidad Mariano Gálvez iarmasg@edu.gt

Guatemala

Fecha de entrega: julio, 2020

Fecha de aceptación: noviembre, 2020

\section{RESUMEN}

Esta investigación tiene como propósito analizar el rol que tienen las emociones en el conflicto escolar en un contexto de postconflicto en Colombia, a partir de las narraciones de los actores educativos. Para el desarrollo de este escrito, se identificaron las situaciones que generan conflicto en el escenario escolar, se estableció la frecuencia que tienen los conflictos, se analizó el papel que tienen las emociones en la generación y en la superación de los conflictos que se presentan en el escenario escolar, se estudiaron las narraciones que hacen los actores educativos de la forma como las emociones intervienen en las situaciones de conflicto y se estableció la incidencia del conflicto escolar en el contexto de postconflicto que vive Colombia actualmente. El estudio se llevó a cabo en el Colegio Miguel Antonio Caro de Engativá y buscó establecer el tipo de situaciones que conducen a la generación de conflicto, la existencia de normas relacionadas con la convivencia y manejo de conflictos en la institución, la agresividad en los conflictos escolares, las formas de superación del conflicto, el nivel de preparación de la institución para superar conflictos, las emociones observadas, las personas emocionalmente involucradas, los hechos generadores de emociones, así como las emociones presentes durante la convivencia pacífica.
\end{abstract}

Palabras clave: conflicto escolar, emociones, postconflicto, narrativas, actores. 


\title{
Analysis of actors narratives towards the role of emotions in school conflict.
}

\begin{abstract}
The purpose of this research is to analyze the role of emotions in school conflict in a postconflict context in Colombia, based on the narratives of educational actors. For the development of this writing, the situations that generate conflict in the school setting were identified, the frequency of conflicts was established, the role that emotions have in the generation and in overcoming the conflicts that arise in the school setting. The narratives made by educational actors of the way emotions intervene in conflict situations were studied, as well as, the frequency of school conflict in the post-conflict context that Colombia is currently under were also established. The study took place at the Miguel Antonio Caro de Engativa High School and it looked to establish the type of situations that lead to the generation of conflict, the existence of norms related to coexistence and conflict management in the institution, aggressiveness in school conflicts, the ways to overcome the conflict, the level of preparation of the institution to overcome conflicts, the emotions observed, the people emotionally involved, the events that generate emotions, as well as, the emotions present during peaceful coexistence.
\end{abstract}

Keywords: school conflict, emotions, post-conflict, narratives, actors 


\section{INTRODUCCIÓN}

Actualmente el tema del conflicto escolar ha cobrado importancia, dada la incidencia que este fenómeno tiene al estar asociado a diversos factores tanto individuales como colectivos y a la violencia generada en la escuela. Es una situación que va en detrimento del respeto de los Derechos Humanos reflejándose en la indiferencia hacia el fomento de una cultura de paz que por consiguiente no hace permisible la armonía, teniendo en cuenta que es en la escuela donde se debe educar para la convivencia, la paz y la civilidad.

Aun así, el conflicto escolar conforma uno de los entornos donde se suscitan situaciones asociadas a conductas de diversa naturaleza entre pares, estudiantes y docentes, cuyas exaltaciones como ira, enfado y mal carácter, no les permite sentirse emocionalmente equilibrados. Por el contrario, se ven involucrados en experiencias que resultan perjudiciales para sí mismos porque causan ansiedad, privaciones, frustraciones, insatisfacciones personales, rechazo por parte de los otros y dañan las relaciones interpersonales. Los jóvenes en edad escolar no han alcanzado un grado de madurez tal que les permita adquirir un mayor conocimiento de sus emociones, ni tener capacidad para identificar aquellas que expresan sus compañeros y docentes, a desarrollar habilidades para controlar las suyas y entender los efectos de las emociones que comúnmente generan desacuerdos y conflictos.

Además, la escuela no se ocupa metódicamente de promover una formación emocional que haga más viable la convivencia, mediante la prevención de situaciones de violencia que puedan ocurrir en el entorno de ésta. Son situaciones cotidianas que generalmente pasan inadvertidas porque "vivimos una cultura que desvaloriza las emociones y no vemos el entrelazamiento cotidiano entre razón y emoción que constituye nuestro vivir humano y no nos damos cuenta de que todo sistema racional tiene un fundamento emocional" (Maturana, 2009, p. 5). Desde esta Perspectiva puede interpretarse la negligencia de la escuela en torno a las emociones y lo que éstas representan no sólo en los procesos de formación individual de los sujetos, sino en el manejo productivo del conflicto. Es precisamente en el entorno escolar donde la cotidianidad lleva a una convivencia que muchas veces genera conflictos o violencia ya sea física o verbal, situaciones que derivan en limitaciones para construir cultura de paz y especialmente respeto a 
los Derechos Humanos.

Esto se vincula a la dinámica institucional, pero su manejo no se ha dado como posibilidad para regular la convivencia como resultado de la resolución del conflicto. Porque quizás no se ha buscado el espacio para establecer entre muchas otras posibilidades, una relación entre emociones como generadoras de ambientes propicios para la vida ciudadana y para el ejercicio de los derechos humanos en la escuela. Se requiere dejar de verlas simplemente como situaciones que erosionan el tejido social.

Las emociones apuntan directamente a evitar o enfrentar peligros, a convertirlos en oportunidades para aprovechar mejor las relaciones con los demás. (Damasio, 2011)

Los trabajos investigativos se revisaron y se clasificaron según las tres categorías implícitas en el tema de investigación. Aquellos que tienen que ver con conflicto, con la convivencia escolar, y con las emociones son los que se describen a continuación.

\section{Conflicto y convivencia escolar}

En primera instancia se tuvo en cuenta el trabajo realizado por Del Rey et al. (2009), quienes manejan un concepto de convivencia con un claro significado positivo y se relaciona con los principios básicos de la educación. Señalan que, sin embargo, cuando se analiza la convivencia, suele hacerse enfocándose en las situaciones problemáticas en lugar de hacerlo en los pilares que la hacen viable, con lo cual se genera una valoración negativa de la escuela que resulta injusta o inapropiada en múltiples oportunidades. El estudio tuvo como propósito comprobar la buena calidad de la convivencia evaluada por la comunidad académica, así como la identificación de puntos clave para la creación de un ambiente favorable a una buena convivencia en la escuela.

Entre los resultados encontrados se menciona que la convivencia escolar es percibida, por los tres colectivos que la integran -educandos, educadores, comunidad de padres-, como una dinámica relacional positiva, aunque en ella existe cierto grado de conflictividad. El aporte de este trabajo a esta investigación, radica en los conceptos sobre convivencia en el ámbito escolar y la forma como ésta contribuye a la convivencia, en la cual el conflicto se hace presente. 
Componentes que tienen la misma dirección de esta idea de investigación.

Un segundo trabajo investigativo que se halló en esta revisión fue el presentado por Garretón (2013), titulado "Estado de la convivencia escolar, conflictividad y su forma de abordarla en establecimientos educacionales de alta vulnerabilidad social de la provincia de Concepción, Chile”. Es una Tesis Doctoral en la cual se señala que la escuela como organización social ha cambiado continuamente en su estructura. Su origen y evolución ha sucedido en paralelo con el desarrollo y evolución de las distintas sociedades y momentos históricos. Antaño, esta era un espacio al que concurría una élite, para recibir instrucción como complemento a la educación que era responsabilidad de la familia.

En la actualidad, la escuela es de carácter obligatorio, lo que ha dado lugar a la interacción de un gran número de personas, desde los docentes y estudiantes y sus respectivas familias, hasta personal auxiliar y de apoyo, que despliegan y se relacionan a través de sus distintos roles, con lo cual construyen día a día un espacio de convivencia que le da a la escuela un sello particular a la institución escolar (UNESCO, 2004). En algunas instituciones educativas el clima de convivencia está basado en el respeto, buenas relaciones, colaboración, y los docentes, estudiantes y familias participan de manera armónica para el logro de los objetivos que esta persigue. También se presentan determinados factores que inciden negativamente en las relaciones interpersonales, e inclusive esos efectos pueden entorpecer el logro de los objetivos institucionales.

Como parte de alcance del estudio, se estudian los significados que puede tener la convivencia escolar, y se identifican algunos de los principales desafíos que pueden afectarla, tales como las manifestaciones agresivas, las expresiones de violencia y los actos hostiles o de acoso entre pares. Así mismo se estudia la correlación que existe entre la vida emocional de los integrantes de la comunidad académica, y la convivencia escolar, con el propósito de establecer si la formación en el manejo de las emociones puede estimular o propiciar climas de sana convivencia. Son aspectos que tienen la misma dirección de esta investigación y por lo mismo se considera que sus lineamientos contribuyen a enriquecer los análisis que aquí se efectúan.

En la misma dirección Romero (2011), en su trabajo sobre la convivencia desde la diversidad, 
da cuenta de una compilación de la fundamentación teórica del programa de Paz y Convivencia, implementando desde el Área de Acompañamiento Integral en todas las sedes de la Universidad Nacional de Colombia. Ese trabajo presenta a la convivencia escolar vista desde el marco de instituciones sociales como los Derechos Humanos y la Cultura de la paz, para lo cual contiene bases teóricas relacionadas con la conceptualización positiva del conflicto y, a su vez, del enfoque pedagógico del manejo de los conflictos. Es una publicación que aporta ideas básicas a todos y cada uno de los miembros de la comunidad, para el abordaje de los procesos de convivencia hacia una comprensión más amplia de situaciones conflictivas propias de la vida académica.

El hallazgo más destacado del estudio hace referencia a que todos los integrantes de la sociedad están obligados a respetar, exigir y velar por los Derechos Humanos, a través de los instrumentos y procesos necesarios dentro de un marco democrático de las instituciones educativas, a las que les compete afrontar el reto de formar integralmente a sus estudiantes, en principios éticos como seres humanos en el contexto de un país afectado históricamente por la violencia. El trabajo se propone tomar los principios constitucionales relacionados con la convivencia pacífica, la realización y la garantía de los derechos, para convertirlos en acciones concretas y tangibles. Con esa visión, el trabajo maneja fundamentos teóricos en Derechos Humanos, conflicto y convivencia que resultan importantes como puntos de referencia para el desarrollo de las temáticas que se trabajan en este caso, que resultan coherentes con las categorías y subcategorías implícitas en el tema del presente estudio.

Otra investigación de carácter socioeducativo es planteada por Cruz (2008) que destaca la gestión y transformación del conflicto por vías pacíficas; es desarrollada en la amazonia colombiana donde el autor encuentra que el conflicto ha hecho presencia con altos niveles de agresividad. Desde esta perspectiva, señala que se requieren procesos educativos para la paz y transformación del conflicto mediante el fortalecimiento de prácticas con grupos sociales que trabajan en la región. La propuesta es una contribución para que los jóvenes mejoren su forma de asumir y tratar el conflicto a través de la empatía, la creatividad y la no violencia en el marco de una educación para la paz.

Su aporte a este trabajo está en el recorrido histórico sobre violencia, conflicto y paz; hace, 
además, una aproximación a la historia de la educación y su aporte a la paz en el contexto mundial, latinoamericano, colombiano y puntualiza en el caso del Caquetá en Colombia. Define igualmente, los fundamentos conceptuales de la violencia, el conflicto y la cultura y educación para la paz; ahonda en el conocimiento de mecanismos formativos y de control que adoptan los centros educativos para hacer frente al fenómeno del conflicto y la forma de tratarlo cuando surge. Concluye la necesidad de emprender acciones y procesos socioeducativos para la construcción de la paz que conozca y reconozca las claves culturales del entorno. Es por los contenidos que hacen referencia a la educación como posibilidad para promover una cultura de paz y convivencia, que se ha tomado en cuenta esta investigación, cuyo direccionamiento teórico y conceptual contribuye a ampliar aquellos que se manejan en el desarrollo de ésta.

Dentro de la misma orientación, Torrego (2006) titula su investigación así: Desde la mediación de conflictos en centros escolares hacia el modelo integrado de mejora de la convivencia: Estrategias de mediación y tratamiento de conflictos; se trata de un trabajo que orienta sobre el manejo del conflicto en la escuela. El autor plantea que, en el entorno de la escuela, la respuesta que se da a los problemas de convivencia puede caracterizarse por la improvisación y la falta de coordinación, manejo que frecuentemente conduce a la adopción de medidas de carácter reglamentario, simples estrategias administrativas que no atacan la verdadera raíz de tales situaciones, con lo cual simplemente se busca evitar problemas para la institución sin que las causas subyacentes sean cuestionadas y menos aún, solucionadas. Ante esta problemática, el principal de los hallazgos es la determinación de la no conveniencia de mantener la inhibición ante el conflicto de convivencia, así como tampoco se puede responder con la represión.

Esta investigación recurre tanto a cuestiones de índole estratégica orientadas al desarrollo profesional del profesorado, como a otras centradas en el contenido de la respuesta educativa a los conflictos de convivencia escolar en general y al comportamiento asocial de los alumnos en particular, tanto desde el punto de vista del currículo escolar como desde el más estrictamente organizativo de los centros de enseñanza. La intención del texto consiste en ofrecer aportes para que los docentes adopten un proceso de autorreflexión, en el que debe partirse de un nuevo 
paradigma en el cual la ausencia de conflictos no es requisito para que pueda haber convivencia pacífica, sino que ésta debe ser la consecuencia de una forma no violenta de resolverlos.

Una vez aceptado ese paradigma, el paso lógico debe consistir en construir en la institución educativa un ambiente en el que el tratamiento prospectivo o propositivo frente a los conflictos, sea la base de la sana convivencia. Ese tratamiento puede incluir tanto medidas preventivas para la resolución pacífica de conflictos por medio de soluciones creativas y respetuosas del otro, como medidas para controlar los conflictos cuyo manejo se haya desbordado hacia actos de violencia. De esta manera, se reconoce en el trabajo que lograr ejecutar esa propuesta de pacífica convivencia en los centros escolares es un reto educativo complejo, ya que indefectiblemente tendrá que ir unida a la vivencia de valores democráticos, como los de justicia, cooperación, respeto a la dignidad y de no violencia.

Se encontró otra investigación de Caballero (2010) sobre convivencia escolar y sus buenas prácticas; se enmarca en la línea de educación para la paz y su finalidad es reconocer algunas especificaciones de prácticas educativas que fomentan la paz y la convivencia en el ámbito escolar, mediante entrevistas estructuradas en diez centros educativos de la provincia de Granada, Red Andaluza "Escuela, Espacio de Paz" sobre la puesta en marcha, desarrollo y evaluación de actuaciones encaminadas a la cohesión del grupo, la educación en valores, la gestión democrática de normas, la regulación pacífica de conflictos y las habilidades de orden socioemocional. A partir del análisis de las entrevistas, los autores del trabajo identifican diferentes actuaciones y medidas que se ejecutan en instituciones que han logrado buenos ambientes de convivencia, a pesar de los inevitables conflictos. Uno de los hallazgos más destacados se refiere a la necesidad de cooperación entre todos los actores que intervienen en el proceso educativo, así como la recomendación de establecer una estrategia formativa de valores asociados a la cultura de paz.

Otra de las investigaciones rastreadas es de autoría de Abate et al. (2008), quienes presentan algunos de los resultados obtenidos en el abordaje de la problemática de la convivencia y violencia en cinco escuelas de San Miguel de Tucumán planteados así: en los últimos años se ha producido un incremento de los hechos de violencia en las escuelas, lo cual implica para docentes y alumnos la construcción de conocimientos significativos y la socialización en la 
interacción conjunta; la convivencia escolar es entendida como la posibilidad de vivir y vivenciar con otros, y el clima institucional de bienestar o de malestar, afecta las relaciones vinculares e incide en el rendimiento académico; la violencia altera la estructura profunda de las instituciones. Concluye que la violencia se presenta como modalidad de relación en las instituciones educativas, en la mayoría de los casos naturalizada, lo que contribuye al debilitamiento de los lazos sociales, sostén esencial para la constitución de sujetos sociales. Se requiere de mayor democratización en las relaciones sociales en la construcción de las normas, en la revisión de la noción de autoridad, justicia y compromiso ético. Valora la importancia del trabajo en grupo y la necesidad de conformar redes solidarias para fortalecer la construcción colectiva de proyectos de convivencia.

Este, como los demás estudios mencionados aportan al desarrollo de la presente investigación, en cuanto comprensión del papel de la escuela en la formación en Derechos Humanos y convivencia en armonía, factores importantes en la educación que se ofrece a los niños, las niñas y los jóvenes, la cual no puede estar desvinculada de la participación de todos los actores de la comunidad educativa, incluyendo, por supuesto, a los padres de familia. Son, además, un precedente importante como reconocimiento del conflicto escolar y su incidencia en la convivencia en entornos educativos, en los cuales se deben fortalecer experiencias comprometidas con la promoción y defensa de los derechos humanos, camino fundamental para el fortalecimiento de la convivencia.

Pérez (2005), entre tanto, se refiere a la conflictividad escolar y el fomento de la convivencia, y realiza diferentes reflexiones en torno a los frecuentes problemas que se pueden presentar entre los alumnos o entre éstos y sus profesores. El autor señala que la convivencia se quebranta frecuentemente en los centros escolares, y puede llegar incluso a impedir la continuación del proceso educativo. Reconocida esa situación de violencia en las aulas, el autor se centra en la identificación de los diferentes problemas existentes, con el fin de comprender mejor esta realidad, y, a partir de esa comprensión, enfocarse en el estudio de posibles vías que conduzcan a su solución. Con ese propósito, en el trabajo se describen algunos de los más frecuentes comportamientos antisociales que tienen lugar en la escuela, y se indican algunos posibles 
cauces para la intervención, como son la disciplina, la mediación y la competencia social. El autor propone una distribución cuatripartita por medio de la cual se organiza la actuación educativa; esa distribución permite avanzar hacia la convivencia en los centros educativos. El autor concluye textualmente que, en su opinión:

Cualquier intervención rigurosa encaminada a remediar los conflictos, más allá de peculiaridades de programas y de técnicas, debe contemplar varios módulos entreverados, a saber: cognitivo, afectivo, ético y social. Esta distribución cuatripartita permite organizar la actuación educativa en la compleja constelación estructural de los comportamientos antisociales, y, en definitiva, avanzar hacia la deseada convivencia. (Pérez V., 2005, p. 52)

Muñoz, Graciano y Ramírez (2017), por su parte, publicaron los resultados de su investigación adelantada en Colombia, en el departamento del Huila, que busca contribuir mediante el registro de experiencias educativas que, como la suya, han logrado mejorar la convivencia escolar en estudiantes del grado sexto en el municipio de San Agustín. Su experiencia se basó en la incorporación de una estrategia didáctica denominada "el teatro foro", la cual permite la creación de espacios de discusión en los que se abre la oportunidad para el debate, la disertación, el empoderamiento y la movilización de pensamiento conducente a la búsqueda de soluciones para superar la problemática de la convivencia escolar estudiantil, tanto en cuanto al clima de aula como al clima familiar. De acuerdo con su experiencia, la práctica pedagógica logró la construcción de estrategias didácticas, generó talleres creativos en los que participaron los estudiantes. Para éstos últimos, la experiencia les mostró una forma de actuar como personas más sociales y humanas mediante una actividad lúdica sencilla y entretenida, que propicia el diálogo entre personas.

A su vez Ortega, Del Rey y Casas (2016) se enfocan en una de las situaciones que con frecuencia conduce a problemas de convivencia escolar, como es el bullying, término que se ha dado en utilizar para referirse a situaciones de intimidación, acoso y malos tratos, y más específicamente se interesa en su prevención. Los autores señalan la escasez de conocimiento sobre la posible existencia de algunos elementos de la convivencia que puedan resultar más relevantes que otros; ese desconocimiento dificulta establecer prioridades para la prevención de 
estas modalidades de conflicto escolar. Ante la situación planteada, los autores exploran lo que denominan valor predictivo de las dimensiones de la convivencia escolar ante la implicación en estos fenómenos. El instrumento empleado es una Escala de Convivencia Escolar (ECE), que fue administrada a 7037 estudiantes (48.9\% mujeres), quienes fueron seleccionados de manera aleatoria entre estudiantes de Educación Secundaria de Andalucía, en España. De acuerdo con ese trabajo, sus autores "destacan la relevancia de las relaciones en el plano vertical y horizontal, particularmente la gestión que realiza el profesorado y los problemas de disruptividad e indisciplina" (Ortega, Del Rey, \& Casas, 2016, p. 99). Advierten, además, que la indisciplina puede considerarse como el problema de convivencia que con mayor frecuencia explica la aparición de problemas de acoso escolar entre iguales y registran que el acoso entre escolares es la expresión más grave de los problemas de conflictividad.

\section{Las emociones y la convivencia escolar}

En relación con el tema de las emociones, igualmente se hallaron algunas investigaciones que ilustran el rol que tienen en un determinado contexto de convivencia. Por ejemplo, Martínez (2014), en su tesis doctoral hace mención de la especial relevancia que se ha reconocido a las emociones y al desarrollo de habilidades para su apropiado manejo, como elemento que puede generar unas competencias superiores en el relacionamiento social de las personas. El trabajo señala que las emociones también pueden ser el reflejo de juicios de valor y creencias del sujeto respecto a lo que para él es importante en su vida. Así mismo, el autor sostiene que la promoción de esas habilidades es cuestión de justicia social.

La investigación se fundamenta en la teoría de Martha Nussbaum (2008), porque señala que las emociones tienen una estructura cognitiva y evaluadora, por lo que un análisis de las emociones permite obtener información sobre el objeto que provoca la intensidad emocional. Evaluar el papel de las emociones es también propósito de la investigación que se propone, al considerar, además, a que se hace referencia a que la intencionalidad de la acción -generada en la emoción, va dirigida siempre a alguien, se deriva de la percepción relevante que le da un sujeto a una situación individual o colectiva según le afecte. De ahí que uno de los hallazgos más destacados de este trabajo esté relacionado con el hecho de que las emociones son determinantes en las interacciones que tienen los sujetos en la vida privada y pública, por lo que se convierten en 
esenciales a la hora de construir ciudadanías democráticas, sensibles a la inclusión de los demás, humanos y no humanos.

De igual manera se descubre que en la comprensión de la cartografía emocional, la descripción de las mismas por parte de los involucrados es determinante para identificar aquellas que se convierten en barreras, así como hay otras que contribuyen a movilizar a las personas para la cooperación y la creación de los proyectos de vida de los sujetos, mientras otras, sólo participan para estigmatizarlos y excluirlos. También se encuentra que fortalecer las emociones, supone reconocer el carácter cognitivo y evaluador que tienen, pero al mismo tiempo confían en que su poder de arrastrar consigo toda la existencia del yo, si se une al pensamiento, será fundamental para la creación de una vida más justa y democrática. De ahí la importancia de educar para la sensibilidad moral.

Seleccionar este trabajo como antecedente del presente, tiene que ver con la similitud de la temática, dado que la meta aquí propuesta se encamina a descubrir en las narraciones de estudiantes y educadores aquellas emociones que en determinado momento de la convivencia en la escuela les genera conflictos. En la dirección, el trabajo de Martínez (2017), cuestiona cuáles son las emociones morales de los estudiantes, que amplían o impiden el interés moral por la vida ciudadana. Hay coherencia en la concepción del sujeto como poseedor de emociones con implicaciones en sus pensamientos, percepciones, acciones que intencionales o no, tienen injerencia en el comportamiento humano.

En coincidencia con estas apreciaciones, se encontró la tesis doctoral de Enríquez (2011), en torno a un programa de regulación emocional basado en la conciencia plena, el cual explica las variables principales que inciden sobre la emoción y relaciona en ese contexto las aportaciones teóricas y experimentales de diferentes autores. El autor sostiene que las emociones son experiencias que involucran de manera simultánea tres diferentes sistemas de respuesta: conductual/expresivo, cognitivo/subjetivo; y fisiológico/adaptativo.

Tras reconocer esas tres dimensiones, se facilita el reconocimiento de las que en determinado momento están determinando la naturaleza de las emociones que emergen en el comportamiento de la persona. 
En el mismo sentido, Chóliz (2005) trata el papel de las emociones en la adaptación del ser humano y explica que es un tema que ha sido ampliamente aceptado y estudiado desde diversos momentos de la historia y por lo mismo desde diferentes puntos de vista desde Hipócrates, Galeno, Darwin, pasando por Le Doux, Cacioppo, Larsen, Smith y Bernston; el concepto llega a la psicología del Renacimiento, encabezada por Robert Burton quien amplió, perfeccionó y elaboró la tesis según la cual la composición de los humores corporales, y en consecuencia el equilibrio anímico del ser humano, es sensible a influencias externas como la alimentación, la edad y las pasiones. Explica el autor que emoción, tal como se concibe actualmente, refleja situaciones de perturbación estímulo que conduce a una respuesta determinada e inconsciente ante un acontecimiento tanto interno como externo. Así mismo reconoce la existencia tanto de emociones voluntarias, como otras innatas, y advierte que las respuestas de evitación se ubican en el punto medio entre estos dos tipos; el autor también reconoce que las emociones son un medio a través del cual es posible establecer cuáles temas o asuntos son de interés o que son significativos para cada persona.

Los tres componentes de la emoción, de acuerdo con este trabajo, son el conductual, el neurofisiológico y el cognitivo. El neurofisiológico se refleja mediante respuestas como taquicardia, vasoconstricción, sudoración, hipertensión, rubor, tono muscular, cambios en los neurotransmisores, sequedad en la boca, respiración, secreciones hormonales, etc. Todas estas son respuestas involuntarias, que no pueden ser controladas de manera voluntaria por el sujeto, aunque son previsibles empleando técnicas apropiadas como puede ser la relajación. Las emociones que experimenta un individuo se pueden inferir a partir de la observación de su comportamiento y de esas respuestas involuntarias, tales como el lenguaje no verbal, las expresiones faciales, el tono de voz, el ritmo, volumen, movimientos del cuerpo, entre otros, aportan señales bastante precisas para un observador experto, y reflejan el estado emocional de la persona.

Al respecto, Damasio (2011) sostiene que las emociones se constituyen de reacciones que promueven la supervivencia de un organismo; tales reacciones pueden asimilarse a las 
expresiones propias del ser humano en respuesta ante una situación del contexto en el que se encuentra. El autor afirma que las emociones propiamente dichas, es decir el miedo, la repugnancia, la tristeza, la felicidad, la vergüenza y simpatía, son mecanismos por medio de los cuales se genera la regulación vital.

En este marco de ideas se inserta el trabajo de Abarca (2003) que corresponde a una tesis doctoral que trata la dimensión emocional en relación con cinco ejes básicos: ¿Cómo surgen las emociones?, las emociones como reacción: reactividad emocional (temperamento), la expresión emocional en los diferentes momentos evolutivos, ¿cómo se desarrolla la conciencia emocional, tanto de las propias emociones como de las emociones de los demás? y ¿cómo se generan los procesos de autorregulación emocional?

Trata igualmente de las dimensiones del desarrollo emocional que se vinculan al desarrollo social, inductoras del desarrollo emocional, y a su vez las competencias emocionales necesarias para un óptimo desarrollo social. Como aspectos determinantes para crear vínculos y desarrollar habilidades sociales, se identifica la regulación de la expresión emocional, la empatía entendida como la capacidad de comprender las emociones ajenas, así como la regulación de las emociones de las personas que rodean al individuo.

Se ocupa además de la socialización emocional en el marco familiar y escolar, en tanto expresión, comprensión y regulación emocional. Expone explicaciones sobre la hipótesis de que, a mayor desarrollo emocional, mejores habilidades sociales y por consiguiente mejor establecimiento de vínculos y experiencias sociales positivas que a su vez incrementan el número de interacciones sociales y estimulan el desarrollo emocional. Destaca cómo las habilidades emocionales contribuyen a alcanzar mayores niveles de satisfacción y desarrollo personal. Expone que ser emocionalmente inteligente consiste en mantener una relación armónica entre las emociones negativas como la ira, la frustración, la ansiedad, los celos, el odio, la frialdad, la arrogancia, la pena, lo que facilita el paso a las emociones positivas como altruismo, alegría, generosidad, humildad y tolerancia.

Los anteriores, son aportes teóricos que dan cuenta de la relación entre emociones y contexto, lo cual interesa en este estudio, ya que tiene que ver con las emociones, generadoras del conflicto que surge en la escuela como consecuencia de la convivencia entre estudiantes y de 
éstos con los docentes, actores principales de las experiencias educativas.

Es allí donde, para indagar, se precisa entender todos los factores que se asocian a las emociones y sus perspectivas en la construcción de subjetividades en un contexto sociocultural como es la escuela.

De otra parte, la Tesis Doctoral de De Souza (2009), profundiza sobre el tema de las competencias emocionales y los conflictos en las escuelas. Toma en cuenta que las emociones y sentimientos median las relaciones interpersonales, lo que la lleva a considerar que el estudio del conflicto interpersonal requiere como primera medida la identificación de las competencias emocionales implicadas en las interacciones. A partir de esa tesis sostiene que la inmadurez emocional, entendida como la carencia de competencias emocionales, conduce a la dificultad para resolver conflictos.

El aporte de ese trabajo incluye la exploración teórica de las competencias emocionales, dado que en el mismo se presenta un recorrido epistemológico de la educación emocional, recorrido que incluye diferentes corrientes de la psicología y la filosofía. Adicionalmente, conceptualiza la inteligencia emocional a partir del análisis de las obras de autores tales como Gardner (1983), Salovey y Mayer (1990) Goleman (1995); el propósito de ese análisis es el de entrelazar diferentes definiciones de la competencia emocional. De esta manera, el trabajo se aproxima a la explicación del conflicto desde diferentes perspectivas, con lo cual logra reconocer la posibilidad del cambio.

Fernández, Ruiz, Salguero, Palomera y Extremera (2017) abordan el tema de la emotividad en el conflicto escolar mediante una metodología cuantitativa, y exploran la relación del Test de Inteligencia Emocional de la Fundación Botín (TIEFBA) con el ajuste personal y escolar de adolescentes españoles. El instrumento empleado se ha basado en el modelo de Mayer y Salovey y evalúa las cuatro ramas mientras que el participante resuelve tareas emocionales generadas por una situación emocional. El TIEFBA fue desarrollado a partir del paradigma del test de juicio situacional e incluye ocho escenas diseñadas con el propósito de evocar emociones positivas y negativas. La muestra analizada incluyó a 1684 adolescentes (48.2\% varones) y obtuvieron mediciones sobre personalidad, empatía, inteligencia y ajuste 
psicosocial. Los autores concluyen que el TIEFBA es una nueva medida prometedora para evaluar la inteligencia emocional, y permitirá a los investigadores y educadores una mejor comprensión de la forma en que la inteligencia emocional afecta a los adolescentes, y evalúa el impacto de las intervenciones.

Este aspecto de la inteligencia emocional también es tema de interés para Elipe, Ortega, Hunter, Simón y Del Rey (2012), aunque lo abordan desde otra perspectiva. Para ellos, la inteligencia emocional es un constructo que, a pesar de que se ha incorporado al campo de la psicología de la educación, ha sido escasamente abordado en el marco de la dinámica social de las aulas escolares. Lo anterior sucede no obstante que, de acuerdo con los autores, estudios previos ponen de manifiesto que el meta-conocimiento sobre las propias competencias emocionales, es un elemento diferencial entre los escolares. El término que emplean es inteligencia emocional percibida (IEP), y puede conducir a que las personas se involucren en conflictos escolares. En su trabajo, profundizan en el análisis de fenómenos de acoso escolar mediados por el uso de las tecnologías de la información y la comunicación, para lo cual emplearon una muestra de 5759 estudiantes adolescentes andaluces. Los resultados obtenidos los llevan a afirmar que, si bien la IEP discrimina el tipo de implicación en el acoso escolar, no sucede lo mismo en el caso del acoso mediado por estas tecnologías.

En un sentido similar, De Ocáriz, Lavega, Mateu y Rovida (2014), destacan que una de las claves de la educación física es la mejora de la convivencia entre los escolares; señalan que la vivencia de emociones positivas o negativas en las situaciones motrices muestra una relación directa con la educación de las relaciones interpersonales. En su estudio se tomó una muestra de 81 estudiantes de Barcelona para examinar la vivencia emocional suscitada por situaciones cooperativas de expresión motriz, por medio de la escala validada de juegos y emociones (GES). Después de cada actividad, los estudiantes anotaron la intensidad experimentada frente a trece emociones y realizaron un comentario sobre las causas que originaron su emoción más intensa.

De acuerdo con los autores, los resultados confirmaron la contribución de la educación física en la vivencia de experiencias positivas. 
Otro trabajo relacionado con los anteriores lo desarrolló Rodríguez (2015), quien se centró en el estudio de la relación que existe entre inteligencia emocional y conflicto en estudiantes de educación básica primaria. El trabajo se desarrolló con niños de una institución educativa de la ciudad de Tunja, capital de Boyacá, en Colombia. La investigación se basó en el empleo de una metodología mixta no experimental, descriptiva. Los estudiantes fueron observados y analizados dentro del aula y se realizó una interpretación y análisis de los datos obtenidos, con el fin de analizar la influencia que puede tener la inteligencia emocional en la resolución pacífica de los conflictos entre niños de grado quinto. Las variables estudiadas fueron capacidad de concentración, interrelación con los compañeros, entorno familiar, comportamientos agresivos, capacidad de estudio y rol del tutor; de acuerdo con las conclusiones del trabajo, estas variables están permeadas por la inteligencia emocional, lo que hace que, al enfrentar un conflicto en el ámbito escolar desde la óptica de las emociones, se forma y se fomenta el enfoque de competencias ciudadanas.

Ortiz, Romera y Ruiz (2017) plantean que el impacto de los contextos en los que tienen lugar las relaciones interpersonales que tejen el escenario de la convivencia escolar se ve influenciado por la competencia individual entre los actores de esas relaciones con el propósito de gestionar sus vínculos interpersonales; así mismo señalan que los hilos que tejen la convivencia en ese ambiente son "la comprensión del punto de vista del otro, el respeto hacia uno mismo y los demás, la regulación de las emociones propias, la comprensión y correcta lectura de las emociones ajenas y la actuación conforme a un criterio moral basado en la tolerancia, la solidaridad y la justicia" (p. 28).

Los factores claves para que la convivencia sea exitosa son la inteligencia emocional, la competencia social y el dominio moral, pues esos factores determinan de manera notoria la manera como se gestionen las relaciones que se establecen con los miembros de la comunidad educativa, así como el estilo empleado para afrontar las situaciones de conflicto y violencia escolar (González, 2015). Como evidencia de ese impacto, se ha logrado establecer que cuando se presentan situaciones bullying "tanto las víctimas como los agresores muestran mayores niveles de atención a sus emociones y menores niveles de regulación emocional que los no 
implicados en esta dinámica violenta” (Ortega, Del Rey, \& Casas, 2016, p. 29).

Roos, Hodges, Peets y Salmivalli (2015) consideran que la ira y las dificultades para controlar los elementos cognitivos y emocionales que influyen en la agresión determinan la forma en que los pensamientos agresivos se transforman en la propia conducta agresiva; existe un proceso mental de planificación o tendencia agresiva que puede conducir a la ejecución de un comportamiento violento que no se concreta cuando se logra ese control.

Las relaciones de convivencia se ven determinadas por la empatía cognitiva, que es la capacidad para considerar los sentimientos de las demás personas con las que se convive; también influye la empatía afectiva, que es la capacidad para vincularse emocionalmente con esas personas.

Es oportuno precisar que la inteligencia emocional implica también las competencias necesarias para el reconocimiento de los sentimientos propios y los ajenos, para motivarse y para manejar apropiadamente tanto las emociones en relación a uno mismo como a los demás; el término puede involucrar aptitudes complementarias, pero diferentes de la inteligencia académica y las habilidades puramente cognitivas que son medidas por el cociente intelectual (Landazabal \& Oñederra, 2010).

De acuerdo con la investigación de Landazabal y Oñederra (2010), los adolescentes que han sufrido reiteradas conductas de intimidación o bullying y los que tienen alto índice general de agresión, coinciden en su bajo nivel de pensamiento constructivo global o inteligencia emocional, lo que los hace poco flexibles, baja capacidad para adaptar su forma de pensar a diferentes situaciones, bajo nivel de aceptación de sí mismo y de los demás, baja capacidad para establecer relaciones gratificantes y escasa tendencia a conceder a otros el beneficio de la duda. También tienen baja emotividad, baja autoestima, baja tolerancia a la frustración y poco optimismo.

Las posturas ideológicas contempladas como antecedentes del problema, conducen a una mejor interpretación del tema de estudio; así, se obtiene un panorama general de la forma como las categorías de investigación han sido tratadas desde otras miradas que permiten una aproximación a la comprensión del mismo. 


\section{MÉTODO}

Se adoptó un enfoque mixto del problema de investigación con el propósito de que las respuestas que se planteen a las preguntas de investigación se puedan generalizar respecto de toda la población objeto de estudio.

El enfoque cualitativo se aplica para la comprensión de las acciones de los sujetos en función de la praxis; concepción desde la cual se cuestionó y explicó la relación entre emociones y conflicto escolar según narraciones de estudiantes y docentes. Esta misma se llevó a la interpretación de sus relatos según la forma en que ellos asuman que las emociones juegan un papel determinado en los conflictos que se experimentan en su cotidiano escolar.

A su vez, el componente cuantitativo se empleó para determinar el tamaño de una muestra que resulte representativa de la población estudiada y para formular los instrumentos de investigación de manera que los hallazgos no se limiten a los sujetos estudiados, sino que conduzcan a la identificación de situaciones comunes a toda la institución que se toma como población. La población objeto de estudio son los 2100 estudiantes de la Institución Educativa Colegio Miguel Antonio Caro de Engativá.

La muestra estuvo integrada por 74 estudiantes de $\operatorname{los}$ grados $8^{\circ}, 9^{\circ}$ y $10^{\circ}$ de la institución; ésta se determina de acuerdo con las recomendaciones de Hernández Sampieri (2010) para la definición de muestras en investigaciones cualitativas y su tamaño, corresponde a un curso de cada uno de los grados mencionados; a su vez, estos cursos se seleccionan teniendo en cuenta que los estudiantes de estos grados, debido a su edad, están en una mayor capacidad de diligenciar los instrumentos de investigación; adicionalmente el hecho que ya hayan superado varios años de su ciclo educativo les permite comprender el proceso de una manera más amplia y tener un mayor conocimiento de diferentes experiencias relacionadas con el conflicto escolar, tanto propias como de sus compañeros, a lo largo de los diferentes grados. De esta manera se 
busca tener una representatividad de las cualidades del problema objeto de estudio en diferentes segmentos de la población, tal como lo recomienda el citado autor.

Las variables principales de esta investigación son las emociones y el conflicto. Aunque el desarrollo conceptual de cada una de estas variables fue objeto del marco teórico del trabajo, con el fin de poder operativizarlas, éstas se definen de manera preliminar a continuación. Los objetivos del trabajo y el diseño de la investigación hacen necesario que estas variables no sean controladas ni manipuladas.

Las emociones pueden definirse como reacciones de orden psicológico y/o fisiológico que se producen como mecanismos de adaptación a ciertos estímulos que recibe el individuo cuando percibe un objeto, persona, lugar, suceso o recuerdo importante (Serrano \& Ibáñez, 2015). A su vez, el conflicto corresponde al conjunto de dos o más situaciones hipotéticas o reales que son mutuamente excluyentes, lo que impide, de manera real o aparente, la posibilidad de que las mismas puedan ser satisfechas de manera simultánea. (Contreras, 2015)

Las dos variables son de naturaleza cualitativa y su medición es nominal. En resumen, las variables se pueden operativizar de acuerdo con la siguiente tabla.

\section{Tabla 1}

Operativización de las variables de investigación

\begin{tabular}{|c|c|c|c|}
\hline Variable & Definición & Naturaleza & $\begin{array}{l}\text { Nivel de } \\
\text { medición }\end{array}$ \\
\hline Emociones & $\begin{array}{l}\text { Reacciones de orden psicológico y/o fisiológico } \\
\text { que se producen como mecanismos de } \\
\text { adaptación a ciertos estímulos que recibe el } \\
\text { individuo cuando percibe un objeto, persona, } \\
\text { lugar, suceso o recuerdo importante }\end{array}$ & Cualitativa & Nominal \\
\hline Conflicto & $\begin{array}{l}\text { Conjunto de dos o más situaciones hipotéticas o } \\
\text { reales que son mutuamente excluyentes, lo que } \\
\text { impide, de manera real o aparente, la posibilidad } \\
\text { de que las mismas puedan ser satisfechas de } \\
\text { manera simultánea }\end{array}$ & Cualitativa & Nominal \\
\hline
\end{tabular}

Fuente: elaboración propia.

Para la recolección de la información se utilizó en primer lugar la observación directa del 
Análisis de las narrativas de actores frente al papel de las emociones en el conflicto escolar

docente investigador, la cual fue registrada en diarios de campo que permitieron registrar el comportamiento de los sujetos de investigación, tanto durante las horas de clase, como durante los horarios de recreación. La información registrada fue la relativa a las situaciones de conflicto y el papel que juegan las emociones durante esas situaciones.

Igualmente se obtuvo información por medio de entrevistas semiestructuradas a estudiantes y docentes. Así mismo se empleó como instrumento la historia oral narrada por los integrantes de la comunidad académica como consecuencia de las entrevistas.

\section{Tabla 2}

Instrumento de entrevista

Análisis de las narrativas de actores frente al papel de las emociones en el conflicto escolar

OBJETIVO: Analizar desde las narraciones de los actores educativos el rol de las emociones en el conflicto escolar en un contexto de postconflicto en Colombia.

\section{Identificación del entrevistado}

Nombre:

Estudiante:

Docente:

\section{Situaciones que catalizan el conflicto en el escenario escolar}

¿Usted diría que en la escuela existe ambiente apto para la convivencia o que prevalece el conflicto? ¿Qué le hace pensar eso?

¿Qué tipo de situaciones son las que conducen a que se genere conflicto en la escuela?

¿Existen normas relacionadas con la convivencia y el manejo de conflictos en la institución? ¿Usted

las conoce? ¿Esas normas son respetadas y utilizadas tal como están diseñadas?

¿Los conflictos que se presentan se manifiestan de manera pacífica o mediante expresiones de agresividad?

¿De qué forma se logran superar las situaciones conflictivas que se presentan para recuperar el ambiente de convivencia?

¿Usted considera que la institución, incluyendo a profesores y estudiantes, está preparada para manejar adecuadamente los conflictos que se presentan?

Papel de las emociones en la generación y en la superación de los conflictos que se presentan en el escenario escolar.

Narraciones que hacen los actores educativos de la forma como las emociones intervienen en las situaciones de conflicto

¿Por qué cree usted que las diferencias que se presentan durante la convivencia en la escuela conducen a que surjan conflictos?

¿Qué actitudes o sentimientos son los que usted cree que están detrás de los conflictos que se presentan en la escuela? Por favor cite ejemplos.

¿A qué cree usted que se deben las reacciones de las personas cuando se presentan situaciones de conflicto? Por favor cite ejemplos.

¿Qué tipo de emociones observa usted durante los conflictos que se presentan en la escuela?

¿Esas emociones incluyen solo a los involucrados directamente en el conflicto o también a los observadores?

¿Qué tipo de emociones cree usted que son las que permiten que se superen los conflictos que se presentan? 
Análisis de las narrativas de actores frente al papel de las emociones en el conflicto escolar

¿Qué hechos o actitudes son los que generan esas emociones que conducen al conflicto?

En los momentos en que no se presentan conflictos, ¿qué tipo de emociones siente usted que se presentan y que permiten la convivencia pacífica?

¿Cómo identifica usted las emociones que tienen las personas que están involucradas en un conflicto?

¿Cómo diferencia esas emociones de las que se presentan cuando la convivencia en la escuela es pacífica?

Fuente: elaboración propia.

El procedimiento para efectuar la interpretación de la información que se recolectó mediante la aplicación de los instrumentos antes mencionados constó de la selección de dicha información, con base inicialmente en el criterio de pertinencia, que se logra al tomar en cuenta solo aquella que se relacionan efectivamente con el tema de investigación. En segunda instancia se tuvo en cuenta el criterio de relevancia, es decir lo que se devela como importante en relación con el tema de investigación y, en tercer término, se hizo la triangulación de la información por cada estamento: educandos y educadores de acuerdo con sus opiniones en relación con los principales tópicos de la investigación.

\section{RESULTADOS}

El 53\% de entrevistados consideran que en la institución sí existe un ambiente propicio a la convivencia; a su vez, el 36\% corresponde al porcentaje de quienes respondieron que el ambiente que se presenta es propicio al conflicto. Hubo $11 \%$ de personas que no se inclinaron por ninguna de estas opciones y señalaron que ambas circunstancias se manifiestan en diferentes momentos y bajo diversas circunstancias.

Las respuestas incluyeron dentro de las causas de conflicto el no ponerse de acuerdo en algún tema o no estar de acuerdo con alguna situación en el salón, las diferencias de personalidad y actos de intimidación. Otros hablaron del hecho de no ponerse en los zapatos del otro, la intransigencia, la falta de comunicación, la falta de tolerancia y de respeto a las opiniones ajenas; otros se refirieron a situaciones de discriminación, ruido, chismes entre estudiantes y disgustos entre ellos, además del desconocimiento o irrespeto de las normas vigentes y la falta de diálogo. 
Hubo algunas respuestas que parecieran buscar justificar el conflicto, al señalar que éste se debe a "constantes faltas de respeto hacia los estudiantes", o "cuando alguien trata mal a otra persona sin razón alguna". Otros dijeron que el conflicto emerge cuando "las personas se ponen muy fastidiosas y quieren ganar atención" y "personas cansonas que no dejan trabajar en clase". Puede notarse que este tipo de respuestas señalan las causas en cabeza de los demás para mostrar el conflicto como algo inevitable; se refirieron a "la mala personalidad de los otros, que hace entre personas haya momentos de un mal ambiente y puede haber posibilidades de generar violencia".

Pudo verse que el $68 \%$ de los encuestados reconoció la existencia de normas para la convivencia y el manejo de conflictos dentro de la institución, lo que claramente indica que tales normas sí existen. No obstante, el 29\% contestó que no existen y 3\% dijo que no lo sabe; este primer hallazgo revela la necesidad de una mayor difusión de estas normas dentro de la comunidad académica, dado que cerca de una tercera parte de los encuestados no las conoce, y eso puede implicar que un considerable porcentaje de los estudiantes, y probablemente también de docentes se comporte como si no existieran, es decir, por lo que son personas propicias a generar conflictos y/o a desconocer el comportamiento que se debe seguir para resolver las situaciones conflictivas; además, son personas que podrían incurrir en sanciones o al menos en comportamientos que alteren la normalidad de las clases y demás actividades instituciones, simplemente por desconocimiento.

Los resultados también mostraron de manera inequívoca que los conflictos que se presentan en la institución son de tipo agresivo en la amplia mayoría de los casos; inclusive varios entrevistados (el 12\%) calificó a esos conflictos como insoportables, mientras que solamente el $3 \%$ los calificó de pacíficos. Es decir que no son solamente intercambios pacíficos sobre sus diferencias de opinión o de puntos de vista, sino que se pasa al terreno de la agresividad y la violencia. A pesar de esa agresividad, las personas en la institución finalmente logran recuperar el control y pasar de una actitud agresiva a una actitud conciliadora, por medio del diálogo o la conversación. Otros entrevistados consideran que la actitud que conduce a resolver los conflictos es la de escuchar, que es uno de los elementos involucrados en el diálogo; en cambio, otros señalan a los orientadores como las personas que finalmente logran que se superen los 
conflictos y otros piensan que tales conflictos realmente no se superan.

Así mismo, el $61 \%$ de las respuestas fueron afirmativas, es decir, que consideran que la institución sí se encuentra preparada para el manejo de conflictos. En principio esta respuesta resulta sorprendente frente a las preguntas precedentes, dado que ya en una respuesta inicial la mayoría de los entrevistados indicaron que los conflictos dentro de la institución son agresivos, lo que resulta extraño en una institución que supuestamente está preparada para superar tal tipo de situaciones. También habían manifestado de manera mayoritaria que no se respetan las normas existentes en la institución para el manejo de conflictos.

Pudo verse que las diferencias de opinión fueron la causa más señalada por los entrevistados para explicar la presencia de conflictos en esta institución. En segundo lugar, aunque con un porcentaje de apenas la mitad que el primero, estuvo la intolerancia y luego, con el 11\%, la complejidad de la convivencia. Es decir que para los entrevistados esos conflictos surgen como una consecuencia de la diversidad de opinión, en un ambiente de intolerancia y que refleja la complejidad de la convivencia que allí se vive a diario.

La emoción más mencionada fue el rencor, que es definido como resentimiento, desazón o queja que queda como consecuencia de un dicho o una acción ofensiva y que puede perdurar por un tiempo más o menos largo y también puede reaparecer cuando se recuerda dicha ofensa (Florencia, 2017); el hecho de que esa fuera la respuesta que mayor participación presentó, hace pensar que los conflictos en la institución pueden ser entre personas que los protagonizan de manera repetida. Esto querría decir que, en la medida en que se dialogue con esas personas y entre esas personas, sería de esperarse que se evite la repetición futura de un número importante de conflictos.

El rencor y la rabia son efectivamente actitudes que desencadenan emociones que conducen al conflicto, por lo que puede afirmarse que los entrevistados son conscientes de que el manejo de las emociones, en este caso, un manejo inadecuado, es el que conduce al conflicto. Si bien se puede buscar que las personas eviten que los demás sientan rencor por cosas que ellos dicen o ira por el trato que reciben, es difícil evitar que alguien, ya no en la institución sino por fuera de ella, incurra en actitudes que conduzcan a esos sentimientos, por lo que se requiere que los integrantes de la comunidad académica aprenda a evitar que sus emociones los lleven a 
situaciones de conflicto que se podrían evitar.

De acuerdo con los resultados el rencor y la rabia juntos son los sentimientos que los entrevistados perciben con mayor frecuencia durante las situaciones de conflicto; se encuentran seguidas por el miedo, la rabia (sin el rencor) y la tolerancia. Estas respuestas indican que las personas en conflicto dentro de la institución estarían manifestando de manera simultánea tanto rencor como rabia, o sea, sentimientos acumulados en el tiempo (rencor), combinados con sentimientos impulsivos (rabia). Se destaca la importancia que los entrevistados le reconocieron al miedo, que es una emoción que se caracteriza por ser desagradable y que normalmente es provocada por la percepción de peligro, real o supuesto, presente, futuro o incluso pasado. (Martínez M. , 2017)

El 87\% de los entrevistados consideró que todos, tanto los directamente involucrados como los observadores, en determinado momento se ven emocionalmente envueltos por los sentimientos que se presentan durante el conflicto dentro de la institución. Este resultado puede indicar que el conflicto no solamente afecta a quienes lo protagonizan, sino a toda la comunidad académica, por lo que las consecuencias no se limitan a las consecuencias emocionales, físicas y académicas que viven quienes se ven involucrados por el conflicto, sino que todas las personas que están en la institución terminan afectadas, lo que indica el alcance que este tipo de situaciones puede acarrear

La expresión más reiterada dentro de los hechos generadores de conflictos se refirió a las tensiones acumuladas por diferentes causas (20\%), seguida por la intolerancia (12\%) y la ira (11\%); el $12 \%$ de los entrevistados señaló varios términos dentro de su respuesta, es decir que consideran que son varios los hechos que, de manera simultánea, generan emociones que llevan al conflicto. En este sentido, la respuesta "varios" puede asimilarse a "tensiones", en la medida en que corresponde a múltiples razones que, sumadas, producen emociones que derivan en conflicto.

En cuanto a las emociones presentes durante la convivencia pacífica, la respuesta que mayor nivel de reiteración tuvo fue la tranquilidad, con el 32\%. Bastante lejos se ubicaron el amor $(14 \%)$ y la tolerancia (12\%); hubo otras respuestas que no se lograron agrupar porque cada una 
fue mencionada un número mínimo de vences inferior al $2 \%$, por lo que se presentan en esta figura como "otras", con el $31 \%$.

La respuesta predominante en relación con las emociones necesarias para la convivencia pacífica fue la que hizo referencia a tranquilidad y armonía, con el 41\%, seguida de tolerancia con $15 \%$. Es decir que se reitera el resultado de la anterior pregunta, según la cual las emociones que permiten la convivencia pacífica concuerdan con las que están presentes una vez se ha consolidado ese tipo de convivencia. Esto quiere decir que la convivencia pacífica es consecuencia y al mismo tiempo es manifestación de que predominen la tranquilidad y la armonía.

Según se indicó, los entrevistados manifestaron mayoritariamente que sí existe un ambiente que propicia la convivencia en la institución, en reconocimiento a los esfuerzos que se hacen desde el momento en que los estudiantes ingresan y se les informa de la existencia del manual de convivencia y de protocolos para manejar conflictos. La convivencia es entendida como la ausencia de agresiones interpersonales físicas y/o verbales; este ambiente es posible gracias a que consideran que no existe hostilidad, que es sustituida por la tranquilidad, aunque también reconocen la presencia de personas que son la excepción al clima predominante. La convivencia es considerada por otros como la capacidad de superar fácil y rápidamente los obstáculos que surgen; sin embargo, también existen personas que citan a los profesores como los responsables de garantizar el ambiente de convivencia.

Así mismo; hay otro grupo de entrevistados que admite la existencia de situaciones de conflicto, las cuales justifican por el carácter complicado de algunos jóvenes, quienes no respetan las normas; otros más, consideran que el conflicto hace parte de la realidad normal o cotidiana que se deriva de las diferencias en los puntos de vista, por lo que puede decirse que lo ven como algo inevitable. También otros identifican que algunas personas dentro de la institución tienen interés en buscar el conflicto como un propósito, a veces como respuesta a las burlas de las que son objeto, lo cual contribuye a que se presente un clima de tensión en el ambiente de la institución.

Igualmente, la falta de condiciones de seguridad es señalada por algunos entrevistados como la causa de los conflictos existentes, además de que ciertos compañeros calificados como 
conflictivos son los que generan esa tensión. Dentro de los estudiantes existen diferentes posturas en relación con la existencia del conflicto, pues mientras unos la reconocen, otros la niegan o la desconocen.

No lograr el acuerdo sobre algún tema, las diferencias de personalidad y los de intimidación son señaladas como las causas del conflicto, así como la falta de empatía, la intransigencia, las debilidades de la comunicación, la intolerancia, la falta de respeto a opiniones de otros, así como manifestaciones de discriminación, rumores o chismes, el irrespeto a las normas vigentes y la falta de diálogo. Algunas respuestas parecieran provenir de quienes justifican el conflicto, pues señalan dentro de los responsables a los malos tratos injustificados, las actitudes fastidiosas, y el deseo de llamar la atención de personas cansonas que impiden el avance normal de las clases.

Fueron señaladas otras causas de conflicto, aunque de manera aislada, como el robo de objetos, envidias, odios, agresiones tanto verbales como físicas y rencores del pasado. Es decir que las causas se pueden agrupar de acuerdo con esas respuestas a unas relacionadas con el carácter de las personas y un segundo grupo de actuaciones de terceras personas.

La mayoría de los entrevistados manifestaron que sí existen normas para la convivencia, aunque la inmensa mayoría reconoció que no tienen aplicación dentro de la institución; en síntesis, las normas existentes carecen del grado de efectividad necesario como para contener el conflicto, tal vez por no contar con un balance de consecuencias suficientemente fuerte para disuadir a quienes generan el conflicto. Los conflictos que se presentan son mayoritariamente caracterizados por agresiones, que van mucho más allá de intercambio de opiniones para convertirse en agresiones y violencia, física o verbal.

No obstante, los estudiantes logran llegar a puntos de diálogo que les permiten superar los conflictos en los que se han enfrascado, a pesar de que, como ya se mencionó, no se aplican las normas previstas por la institución para el manejo de estas situaciones. De manera paradójica, los entrevistados respondieron que la institución sí se encuentra preparada para manejar las situaciones de conflicto, lo que, de acuerdo con las respuestas previas, implica que ese manejo se logra por fuera de los mecanismos previstos oficialmente por la misma institución. 
Las diferencias en las opiniones frente a los asuntos cotidianos fueron señaladas como la principal razón que conduce a conflictos; esto unido a la falta de tolerancia en un ambiente en que la convivencia por si misma es reconocida como algo complejo. Y las actitudes percibidas como las que más generan conflicto fueron el rencor y la rabia, es decir, actitudes negativas hacia los demás, ya sea de manera prolongada en el tiempo o como una reacción momentánea ante la percepción de un trato injusto. Estos hallazgos suponen diferentes técnicas para el manejo de los conflictos, dado que incluyen a personas que los generan de manera reiterada, y de actitudes de baja tolerancia que pueden aprender a manejarse por medio del control de las emociones, como lo reconocen los mismos entrevistados.

Durante los momentos de conflicto, los entrevistados perciben situaciones de odio y rabia, entre otras, así como miedo que finalmente los lleva a encontrar soluciones con los compañeros con quienes están en conflicto. Así mismo los entrevistados reconocen que estas emociones no solamente implican a los involucrados de manera directa en las situaciones de conflicto, sino también a los observadores, por lo que las consecuencias indeseables del conflicto pueden llegar a extenderse a todos los miembros de la comunidad educativa. Las tensiones, la intolerancia y la ira son las circunstancias y emociones que conducen al conflicto, de acuerdo con los resultados de las encuestas, por lo que se puede suponer que la generación de espacios de esparcimiento puede contribuir a que las tensiones entre estudiantes se alivien y se prevengan conflictos de esa manera.

También manifestaron los estudiantes que la tranquilidad es el tipo de emoción que predomina cuando no hay conflicto, en momentos de plena convivencia; esa misma sensación es la que conduce a prevenir que los conflictos emerjan entre los estudiantes.

Al confrontar estos resultados con el marco teórico previamente desarrollado se generan diversos hallazgos que enriquecen el aprendizaje producido por la presente investigación, los cuales se mencionan a continuación. Se confirma que, como lo advirtió Nussbaum (2008), los integrantes de la comunidad académica tienen la opción de ser capacitados para generar respuestas inteligentes ante las situaciones que hasta el momento no saben cómo controlar, por lo que desbordan en forma de situaciones conflictivas.

Se necesita entonces lograr que estas personas adquieran un manejo cada vez más consciente de 
la forma como las emociones en la actualidad terminan dominándolos, pero se trata de una situación que es susceptible de modificarse de manera consciente. El miedo fue una de las emociones mencionadas por los estudiantes durante las situaciones de conflicto, la cual concuerda con la postura de Damasio (2011), como un mecanismo de regulación vital que les permite evadir situaciones de riesgo a los que son llevados debido precisamente a que desconocen la forma de controlar sus emociones. Los estudiantes se están dejando llevar por las emociones como consejeras indeseables de su comportamiento.

Tales actitudes de los estudiantes corresponden a lo que Piqueras, Ramos, Martínez y Oblitas (2009) denominan como estimulaciones fisiológicas que constituyen emociones básicas o primarias, contrarias a aquellas más maduras o inteligentes que se pueden alcanzar cuando obedecen a un proceso de aprendizaje y conocimiento de sí mismos. Según Puente (2007), las emociones que llevan al conflicto son pasajeras, a pesar de que pueden ser muy intensas, por lo que pueden superar con facilidad a personas que no hayan sido formadas o capacitadas para reconocerlas a tiempo para poder controlarlas antes de que les causen daño en sus relaciones con los demás.

La educación en el manejo de las emociones es el camino señalado por Bisquerra (2001) para el control de esos impulsos emocionales que conducen al conflicto, según lo reconocen también los entrevistados en esta investigación. Las reacciones involuntarias o reflejas, casi de carácter instintivo pueden ser sustituidas por reacciones pensadas, razonadas o inteligentes que tengan en cuenta la naturaleza humana y se basen en el carácter de quien las domina de manera consciente o racional.

Temor, intranquilidad, ira, hostilidad, fueron emociones reconocidas por los entrevistados, lo cual concuerda con el planteamiento de Casassus (2006). Tales emociones pueden ser sustituías por respuestas de tipo vigilado o racional que pueden tomar los estudiantes, de acuerdo con Pérez y Redondo (2006). Empleando la propuesta teórica de Casassus (2006), puede notarse que estos estudiantes están actuando de forma inconsciente, por lo que les asignan a los hechos que se les presentan una valoración superior a la que en realidad tienen. El rencor y la rabia que motivan sus reacciones se deben a que sienten que han sido objeto de burla, la cual puede en realidad tratarse de una provocación que sus compañeros generan al saber que ellos son 
incapaces de controlar de manera consciente las respuestas que dan a los hechos que observan.

Partiendo de ese diagnóstico, se puede aceptar la recomendación de García (2012) para que los estudiantes adquieran nuevos conocimientos que les ayuden a reconocer, diferenciar, controlar y filtrar las sensaciones que captan sus sentidos y que en su mente se transforman en emociones que actualmente pasan de manera automática a convertirse en respuesta, sin ninguna mediación de la consciencia.

\section{DISCUSIÓN Y CONCLUSIONES}

De acuerdo con los hallazgos que generó la investigación de campo, se encontró que no ponerse de acuerdo en algún tema o no estar de acuerdo con alguna situación en el salón, las diferencias de personalidad, así como los actos de intimidación son las situaciones que con mayor frecuencia generan conflicto en el escenario escolar.

Adicionalmente, la falta de empatía, la intransigencia, las fallas en la comunicación, la intolerancia y la falta de respeto ante las opiniones ajenas también contribuyen o conducen a situaciones de conflicto, así como casos de discriminación, rumores y chismes entre estudiantes, al igual que desconocimiento o irrespeto de las normas vigentes y la falta de diálogo.

Determinados entrevistados plantearon respuestas que pueden interpretarse como tolerantes de causas generadoras de conflicto, por lo que se identifican con personas que probablemente protagonizan ese tipo de comportamientos. Específicamente se refirieron a constantes faltas de respeto hacia los estudiantes, o los tratos inadecuados que se presentan sin ninguna razón que los justifique. Igualmente señalaron en esas respuestas que el conflicto surge cuando "las personas se ponen muy fastidiosas y quieren ganar atención" o cuando se trata de personas que fastidian tanto que logran interrumpir el avance normal de las clases. Estas últimas corresponden a respuestas que colocan las causas en cabeza de los demás, por lo que se trata, supuestamente, de circunstancias que se salen de control de quien está contestando de esa manera, a manera de justificación.

Hubo una frecuente alusión a la rabia, la burla, y la supuesta "buena" intención de defender a 
un compañero, además de ira, rivalidad y rencor. Existen otras causas de menor incidencia en el conflicto, como el robo de objetos, al igual que sentimientos como envidia y odio, agresiones verbales y físicas, intimidación o algunas discusiones pasadas de tono. Así mismo, surgen retos entre compañeros que terminan en conflicto e incluso casos de tráfico de drogas.

Varias de estas respuestas hacen referencia a aspectos relacionados con el carácter de las personas, lo que hace que las situaciones cotidianas se conviertan en conflicto; un segundo grupo de respuestas que se centra en las actuaciones o actitudes que son presentados como justificaciones de la existencia del conflicto.

Dentro de las emociones que fueron mencionadas como originadoras del conflicto, los entrevistados mencionaron el rencor, que entendido como resentimiento que queda como consecuencia de un dicho o una acción ofensiva; este sentimiento puede perdurar un tiempo y también puede reaparecer cuando la personas recuerda la ofensa. Este hallazgo conduce a pensar que los conflictos en la institución pueden ser entre personas que los protagonizan de manera recurrente.

También se mencionaron la rabia y la intolerancia; la primera corresponde a un sentimiento que emerge cuando una persona toma la decisión consciente de actuar para detener de manera inmediata el comportamiento amenazante de otra fuerza externa; se experimenta cuando alguien percibe que está siendo tratado de maneja injusta. Se trata entonces de una reacción voluntaria, en la medida en que la persona esté capacitada para controlar sus impulsos; una reacción de corto plazo, casi instantánea, lo que implica que la voluntad debe mediar de manera rápida para impedir que la persona actúe de manera incontrolada, llevada por esa emoción.

El hecho de que el rencor y la ira sean emociones que de manera recurrente conducen a situaciones de conflicto en esta institución implica que tales conflictos se caracterizan por la reiteración, por lo que su frecuencia es alta. Cuando las personas logran que el rencor no sea el que los lleve a situaciones conflictivas, logran distanciar el estímulo de la respuesta, por lo que sería menos probable que se presenten tales situaciones repetitivas. Así mismo, cuando las personas son capaces de impedir de actuar movidas por el impulso repentino de la rabia, es menos probable que una rabia transitoria termine convirtiéndose en el motor de actos de 
conflicto. En cambio, personas que carecen de recursos psicológicos necesarios para controlar los efectos de estas emociones, seguramente incurren en conflictos de manera permanente y descontrolada.

A pesar de ello, algunos estudiantes señalaron que la institución cuenta con las herramientas necesarias para controlar los conflictos, a pesar de que en su mayoría los entrevistados manifestaron que las normas existentes para estos casos no son respetadas.

Los hallazgos mostraron que las emociones juegan un papel determinante en el surgimiento de las situaciones de conflicto. En concordancia con el análisis que a nivel teórico han hecho los diversos autores citados en este trabajo, los resultados de la investigación de campo indican que las emociones y especialmente la falta de control consciente de ellas, es determinante de las situaciones de conflicto.

El rencor, la rabia, la ira, la intolerancia, la sensación de burla, de maltrato y desconocimiento de las normas son el alimento del que se nutren las situaciones de conflicto que los entrevistados señalaron con mayor frecuencia. En contraste con ellas, la tolerancia y la tranquilidad no solamente caracterizan las situaciones de convivencia, sino que además las prolongan en el tiempo y ayudan a evitar la presencia de situaciones de conflicto.

Se destaca la concordancia que existe entre las narraciones de los entrevistados y las propuestas teóricas que los múltiples autores citados han alcanzado en relación con la forma en que las emociones determinan la presencia de situaciones de conflicto.

En efecto, los integrantes de la comunidad académica pueden ser capacitados para lograr la generación de respuestas inteligentes ante las situaciones que hasta el momento no saben cómo controlar, y de esa forma reducir la frecuencia y la intensidad de las situaciones de conflicto. Se trata de que los integrantes de la comunidad educativa alcancen un manejo cada vez más consciente de la forma en que sus propias emociones pueden dominarlos, y que esa situación se puede modificar de manera consciente. Ya los encuestados manifestaron que el miedo también está presente las situaciones de conflicto, lo que revela que ellos temen a las consecuencias indeseables del conflicto.

Se trata de estimulaciones fisiológicas que hacen parte de emociones básicas o primarias, que pueden ser sustituidas por otras más maduras o inteligentes que pueden ser alcanzadas cuando 
corresponden al resultado de proceso de aprendizaje y autoconocimiento. Esas emociones que conducen al conflicto son pasajeras, aunque pueden ser muy intensas; también pueden ser reconocidas de forma oportuna cuando la persona ha recibido el entrenamiento necesario, con lo que se evitaría terminen convirtiéndolos en sus propias víctimas debido a las mañas relaciones con los demás.

La educación en el manejo de las emociones lleva al control de esos impulsos emocionales generadores de conflicto. Las reacciones involuntarias o reflejas, de tipo instintivo pueden ser sustituidas por otras racionales o inteligentes que consideren la naturaleza humana. De esa manera, emociones como temor, intranquilidad, ira y hostilidad, entre otras, pueden ser reemplazadas por respuestas vigiladas o racionales; en la actualidad los estudiantes actúan de forma inconsciente, por lo que valoran las situaciones que presencian con una escala que las hace ver con mayor relevancia de la que en realidad les corresponde. Con frecuencia se perciben como a sí mismos como objetos de burla, cuando realmente podría tratarse de provocaciones conscientes de sus compañeros.

El hecho de que los estudiantes en la actualidad sigan actuando de manera no racional ante situaciones cotidianas que llevan a que sus emociones se desborden y terminen en conflictos con sus compañeros, es un síntoma que genera preocupación en un escenario de postconflicto como el que enfrenta el país en este momento. Jóvenes que actúan de esa forma no se encuentran aún preparados para enfrentar de manera exitosa los retos que supone la superación efectiva de la fase actual de postconflicto.

Este trabajo constituye un diagnóstico que muestra un síntoma preocupante de intolerancia y falta de formación para el control de las emociones, capaz de lograr que las personas logren ver las situaciones con un poco más de distancia, de manera que logren apartarse de situaciones violentas. 


\section{REFERENCIAS}

Maturana, H. (2009). Emociones y lenguaje en educación y política. Santiago de Chile: Dolmen.

Damasio, A. (2011). En busca de Spinoza. Neurología de la emoción y los sentimientos. Barcelna: Ediciones Destino.

Del Rey, R., Ortega, R., \& Feria, I. (2009, Departamento de Psicología Universidad de Córdoba). Convivencia escolar: fortaleza de la comunidad educativa y protección ante la conflictividad escolar. Retrieved 2015 from http://www.aufop.com/

Garretón, P. (2013). Estado de la convivencia escolar, conflictividad y su forma de abordarla en establecimientos educacionales de alta vulnerabilidad social de la provincia de Concepción, Chile. Concepción, Chile: Facultad De Ciencias de la Educación Departamento de Psicología. Tesis doctoral.

UNESCO. (2004). Los aprendizajes de los estudiantes de América Latina y el Caribe. (Primer reporte del Segundo Estudio Regional Comparativo y Educativo, SERCE. Santiago de Chile: UNESCO.

Romero, F. (2011). La convivencia desde la diversidad. Bogotá: Universidad Nacional de Colombia. Dirección Nacional de Bienestar.

Cruz, F. (2008). Educar para gestionar conflictos en una sociedad fragmentada. Una propuesta educativa para una cultura de paz. Bacelona: Universidad de Barcelona, Departamento de Teoría e Historia de la Educación. Tesis de Doctorado en Historia de la Educación.

Torrego, J. (2006). Desde la mediación de conflictos en centros escolares hacia el modelo integrado de mejora de la convivencia Estrategias de mediación y tratamiento de conflictos. Barcelona: Graó.

Caballero, M. (2010). Convivencia Escolar. Un estudio sobre buenas prácticas. Revista Paz y Conflictos. Universidad de Granada, 10-20.

Abate, N., Zabala, A., \& Garrocho, M. (2008). La violencia en la escuela: las voces de directivos, docentes, y alumnos. Buenos Aires: XV Jornadas de Investigación y Cuarto Encuentro de Investigadores en Psicología del Mercosur. Facultad de Psicología.

Pérez, V. (2005). Conflictividad escolar y fomento de la convivencia. Revista iberoamericana de educación, 33-52. 
Muñoz, M., Graciano, W., \& Ramírez, V. (2017). Mejoramiento de la convivencia escolar en el grado sexto a partir del teatro foro como estrategia didáctica. Avances en liderazgo y mejora de la educación. Rilme , 326-332.

Ortega, R., Del Rey, R., \& Casas, J. (2016). La Convivencia Escolar: clave en la predicción del Bullying. Revista Iberoamericana de Evaluación Educativa , 91-102.

Martínez, E. (2014). Base Emocional de la Ciudadanía. Narrativas de Emociones Morales en Estudiantes de Noveno Grado de Dos Instituciones Escolares de la Ciudad de Bogotá. Bogotá: Universidad de Manizales-Cinde. Doctorado en Ciencias Sociales: Niñez y Juventud.

Martínez, M. (2017). Coraza de miedo y ego. Medicina Narrativa, 7(2) , 146-148.

Enríquez, H. (2011). Inteligencia Emocional Plena: Hacia un Programa de Regulación Emocional Basado en la Conciencia Plena. Málaga, España: Universidad de Málaga, Facultad de Psicología. Tesis Doctoral.

Chóliz, M. (2005). Psicología de la emoción: el proceso emocional. Retrieved 2015 from www.uv.es/=choliz

Abarca, M. (2003). La educación emocional en la Educación Primaria: Currículo y Práctica. Barcelona: Universidad de Barcelona. Tesis Doctoral.

De Souza, L. (2009). Competencias emocionales y resolución de conflictos interpersonales en el aula. Barcelona: Universidad Autónoma De Barcelona. Programa de Doctorado: Educación y Sociedad. Tesis Doctoral.

Fernández, P., Ruiz, D., Salguero, J., Palomera, R., \& Extremera, N. (2017). La relación del Test de Inteligencia Emocional de la Fundación Botín (TIEFBA) con el ajuste personal y escolar de adolescentes españoles. Revista de Psicodidáctica , 1-8.

Elipe, P., Ortega, R., Hunter, S., \& del Rey, R. (2012). Inteligencia emocional percibida e implicación en diversos tipos de acoso escolar. Psicología Conductual , 169-181.

De Ocáriz, U., Lavega, P., Mateu, M., \& Rovida, G. (2014). Emociones positivas y educación de la convivencia escolar. Contribución de la expresión motriz cooperativa. Revista de Investigación Educativa, 309-326.

Rodríguez, A. (2015). Inteligencia emocional y conflicto escolar en estudiantes de Educación Básica Primaria. Una experiencia desde el contexto de aula. Revista de Ciencias Sociales , 53-72. 
Ortiz, O., Romera, E., \& Ruiz, R. (2017). La competencia para gestionar las emociones y la vida social y su relaión con el fenómeno del acoso y la convivencia escolar. Revista Interuniversitaria de Formación del Profesorado, 27-38.

González, V. (2015). Las habilidades sociales en los fenómenos de violencia y acoso escolar. Bogotá: UNAD.

Roos, S., Hodges, E., Peets, K., \& Salmivalli, C. (2015). Anger and effortful control moderate aggressogenic thought-behaviour associations. Cognition and Emotion, 1-9.

Landazabal, M., \& Oñederra, J. (2010). Inteligencia emocional en las víctimas de acoso escolar y en los agresores. European journal of education and psychology, 243-256.

Hernández Sampieri, R. (2010). Metodología de la Investigación. Bogotá: McGraw Hill.

Serrano, M., \& Ibáñez, M. (2015). La prevención de las situaciones de impacto emocional en el ambiente laboral: Estudio teórico y análisis de su incidencia en el personal sanitario de la comunidad autónoma de Euskadi-España. Trabajo y sociedad, 24, 436-492.

Contreras, R. (2015). Conflicto. Ecunomía. Revista en Cultura de la Legalidad, 212-221.

Florencia, J. (2017). El rencor y otros filos. La Colmena, (11) , 58-64.

Nussbaum, M. (2008). Paisajes del Pensamiento. La inteligencia de las emociones. Barcelona: Paidós.

Piqueras, M., Ramos, V., Martínez, A., \& Oblitas, L. (2009). Emociones negativas y su impacto en la salud mental y física. Suma Picológica , 85-112.

Puente, A. (2007). Cognición y aprendizaje. España: Fundamentos psicológicos.

Bisquerra, R. (2001). Educación emocional y bienestar (1a. reimpresión) (2da. edición). Barcelona: CISS Praxis.

Casassus, J. (2006). La educación del ser emocional. México: Ediciones Castillo.

Pérez, M., \& Redondo, M. (2006). Procesos de valoración y emoción: Características, desarrollo, clasificacación y estado actual. Revista electrónica de motación y emoción, 22.

García, J. (2012). La educación emocional, su importancia en el proceso de aprendizaje. Revista Educación , 97-109.

Castillo, Y. (2012). Factores asociados a la resistencia de los docentes al uso de las TIC en la Facultad de Ciencias Naturales, Exactas y Tecnología de la Universidad de Panamá. 
ICASE, Unidad de Investigación y Tecnología. Panamá: Taller del ICASE. Panamá: Taller del ICASE.

Castillo, Y. (2009). Nivel de conocimientos acerca de las TIC que tienen los docentes de la Facultad de Enfermería- Universidad de Panamá. Panamá: Taller del ICASE.

Castillo, Y., Castillero, A., \& Welsh, G. E. (2014). Usos que dan a las TIC en los procesos de aprendizaje los estudiantes de la Universidad de Panamá. Panamá: Universidad de Panamá.

Panamá, U. d. (2013). Plan de desarrollo institucional. Panamá.: Universidad de Panamá.

Salina, J. (2004). Innovación docente y uso de las TIC en la enseñanza universitaria. Revista Universidad y Sociedad del Conocimiento , 1, 16.

Welsh, G., Castillo, Y., \& Castillero, A. (2014). Percepción de los estudiantes de primer ingreso a la Universidad de Panamá acerca de sus conocimientos y uso de las Tecnologías de la Información y de la Comunicación. Panamá: Taller del ICASE.

Jacobs, H. H. (2014). Curriculum XXI. España Madrid: Narcea, S.A. de Ediciones.

Sevillano García, M. L. (2008). Nueva Tecnologías en Educación social. España Madrid: McGraw-Hill.

Sacristán, A., Cassany, D., Fretes, G., Knobel, M., Lankshear, C., Julio, M., et al. (2013). Sociedad del Conocimiento, Tecnología Educación. Madrid: Morata, S.L. 